\title{
Numerical Simulation of a Linear Stochastic Oscillator with Additive Noise
}

\author{
Aslaug H. Strømmen Melbø $\varnothing^{*} \quad$ Desmond J. Higham ${ }^{\dagger}$
}

Second Revision for Applied Numerical Mathematics, January 2004

\begin{abstract}
The ability of numerical methods to reproduce long-time features of a linear stochastic oscillator are examined. It is shown that certain, widelyused, methods fail to capture the correct second moment growth rate, whereas a customized extension of the partitioned Euler method behaves well in this respect. It is also shown that the partitioned Euler method inherits an infinite-oscillation property. A weaker oscillation result is proved for a wide class of numerical methods.
\end{abstract}

\section{Introduction}

This work is concerned with long-time numerical simulation of a linear stochastic oscillator. Although finite-time convergence theory for globally Lipschitz stochastic differential equations (SDEs) is well established, [5], far less is known about the effectiveness of numerical methods over long time intervals. By focussing on a linear oscillator we are able to obtain precise results about the ability of numerical methods to preserve the properties of (a) having linear growth in the second moment and (b) oscillating infinitely often. In particular, we show that

*Formerly: Department of Mathematical Sciences, Norwegian University of Science and Technology, 7491 Trondheim, Norway. Presently: WesternGeco Oslo Technology Center, P.O.Box 234, 1372 Asker, Norway.

${ }^{\dagger}$ Department of Mathematics, University of Strathclyde, Glasgow G1 1XH, UK. Supported by a Research Fellowship from The Leverhulme Trust. 
the standard Euler-Maruyama method can be greatly improved at no extra computational cost. This conclusion is consistent with that in [8, Proposition 6.2], where mean-square error estimates for long time intervals were derived.

In section 2 we introduce the linear stochastic oscillator and state its second moment and oscillation properties. Both properties relate to infinite time intervals, and hence the traditional finite-time convergence theory for numerical methods applied to SDEs does not automatically guarantee that these features will be reproduced numerically. In section 3 we show that the Euler-Maruyama method does not maintain the linear growth property but, rather, produces a second moment that increases exponentially with time (Theorem 3.1). By contrast, we also show that an implicit, backward Euler-Maruyama method has second moment bounded above for all time. Motivated by the Partitioned Euler method, which has proved successful in the simulation of deterministic Hamiltonian problems, we then introduce a Partitioned Euler-Maruyama method that gives linear growth for all stepsizes less than 2 , and at a rate that is asymptotically exact as the stepsize tends to zero (Theorem 3.3). We show in section 4 that the Partitioned Euler-Maruyama method inherits a precise analogue of the infinite oscillation property (Theorem 4.1). In section 5 we give a weaker but more widely applicable oscillation result. We show that strong finite-time convergence is enough to ensure that given any $\epsilon>0$ and $N>0$, by choosing a sufficiently small stepsize an Itô-Taylor method will pass within $\epsilon$ of the origin at $N$ distinct time points with probability greater than $1-\epsilon$ (Theorem 5.1). The proof of this theorem relies on a lemma that bounds the probability of the first zero-crossing time of the exact solution exceeding some value $T$ independently of the initial data (Lemma 5.1).

\section{Stochastic Oscillator}

A linear stochastic oscillator with additive noise can be written $\ddot{x}(t)+x(t)=$ $h \dot{W}(t)$, or, more precisely, as the two-dimensional stochastic differential equation

$$
\begin{aligned}
d x(t) & =y(t) d t, \\
d y(t) & =-x(t) d t+h d W(t),
\end{aligned}
$$

where $h>0$ is a constant and $W(t)$ is a standard Wiener process. For initial data $x(0)=x_{0} \in \mathbb{R}, y(0)=y_{0} \in \mathbb{R}$, it can be shown [6] that this equation has the unique solution

$$
\begin{aligned}
& x(t)=x_{0} \cos t+y_{0} \sin t+h \int_{0}^{t} \sin (t-s) d W(s), \\
& y(t)=-x_{0} \sin t+y_{0} \cos t+h \int_{0}^{t} \cos (t-s) d W(s) .
\end{aligned}
$$


As in $[6,7]$, for definiteness, we will focus on the case where $x_{0}=1$ and $y_{0}=0$. We are interested in long-time behaviour of the solution, and we will look at the two properties given by the following theorems.

Theorem 2.1. For the linear stochastic oscillator (1)-(2) with $x_{0}=1, y_{0}=0$, the second moment of the solution satisfies $\mathbb{E}\left[x(t)^{2}+y(t)^{2}\right]=1+h^{2} t$.

Proof. The result follows directly from (3)-(4).

Theorem 2.2 (Markus and Weerasinghe). For the linear stochastic oscillator (1)-(2) with $x_{0}=1, y_{0}=0$, almost surely, $x(t)$ has infinitely many zeros, all simple, on each half line $\left[t_{0}, \infty\right)$ for every $t_{0} \geq 0$.

Proof. See [7, Theorem 3] or [6, Theorem 4.1, Chapter 8].

\section{Second Moment Properties of Euler-based Schemes}

In the deterministic setting, it is well known that numerical methods do not automatically inherit long-time behavior from an underlying differential equation. In particular, with $h=0$ in (1)-(2), Euler's method incorrectly spirals outwards, and the backward Euler method incorrectly spirals inwards, whereas a carefully chosen symplectic method will remain on the manifold $x(t)^{2}+y(t)^{2}=$ constant, $[2,12]$. In this section, we develop simple extensions of these results for the linear stochastic oscillator. The analysis is in the same spirit as previous work on mean-square linear stability (for example, $[3,11]$ ) but we note that the noise term considered here is additive rather than multiplicative. Related asymptotic results for moments and stationary laws have appeared in [13, 14].

Applying a numerical timestepping method to the problem (1)-(2) produces discrete approximations $\left\{x_{n}\right\},\left\{y_{n}\right\}$ with $x_{n} \approx x\left(t_{n}\right)$ and $y_{n} \approx y\left(t_{n}\right)$. We assume that a constant stepsize, $\Delta t$, is used, so that $t_{n}=n \Delta t$. We are concerned with the regime where $\Delta t>0$ is fixed, and $n \rightarrow \infty$, so $t_{n} \rightarrow \infty$. The simplest and most widely used numerical method is Euler-Maruyama (EM) [5], which gives the recurrence

$$
\begin{aligned}
& x_{n+1}=x_{n}+\Delta t y_{n}, \\
& y_{n+1}=y_{n}-\Delta t x_{n}+h \Delta W_{n}, \quad \text { where } \Delta W_{n}=W\left(t_{n+1}\right)-W\left(t_{n}\right) .
\end{aligned}
$$

Theorem 3.1. Consider the recurrence (5)-(6) arising from the Euler-Maruyama method applied to the linear stochastic oscillator (1)-(2) with $x_{0}=1, y_{0}=0$. For any $\Delta t \leq 2$,

$$
\mathbb{E}\left[x_{n}^{2}+y_{n}^{2}\right] \geq e^{\left(\frac{1}{2} \Delta t\right) t_{n}}
$$


Proof. Squaring (5) and (6), adding and taking expected values, using the facts that $\Delta W_{n}$ is independent of $x_{n}$ and $y_{n}$ and $\mathbb{E}\left[\Delta W_{n}^{2}\right]=\Delta t$, we find that

$$
\mathbb{E}\left[x_{n+1}^{2}+y_{n+1}^{2}\right]=\left(1+\Delta t^{2}\right) \mathbb{E}\left[x_{n}^{2}+y_{n}^{2}\right]+h^{2} \Delta t \geq\left(1+\Delta t^{2}\right) \mathbb{E}\left[x_{n}^{2}+y_{n}^{2}\right]
$$

Hence, $\mathbb{E}\left[x_{n}^{2}+y_{n}^{2}\right] \geq\left(1+\Delta t^{2}\right)^{n} \geq e^{\left(\frac{1}{2} \Delta t\right) t_{n}}$.

Theorem 3.1 shows that given arbitrarily small $\Delta t>0$, EM produces solutions with second moment that grows exponentially with $t_{n}$, a rate that is qualitatively different from the linear growth rate for the true second moment (Theorem 2.1).

Next we look at the backward Euler-Maruyama (BEM) method, which is also known as the implicit Euler-Maruyama method, see, for example [5, Chapter 12]. Applied to (1)-(2) this gives

$$
\begin{aligned}
& x_{n+1}=x_{n}+\Delta t y_{n+1}, \\
& y_{n+1}=y_{n}-\Delta t x_{n+1}+h \Delta W_{n}, \quad \text { where } \Delta W_{n}=W\left(t_{n+1}\right)-W\left(t_{n}\right) .
\end{aligned}
$$

Theorem 3.2. The recurrence (7)-(8) arising from the backward Euler-Maruyama method applied to the linear stochastic oscillator (1)-(2) with $x_{0}=1, y_{0}=0$ produces

$$
\mathbb{E}\left[x_{n}^{2}+y_{n}^{2}\right] \leq 1+\frac{h^{2}}{\Delta t}, \quad \text { for all } n \geq 0 \text {. }
$$

Hence,

$$
\lim _{t_{n} \rightarrow \infty} \frac{\mathbb{E}\left[x_{n}^{2}+y_{n}^{2}\right]}{t_{n}}=0
$$

Proof. The relations (7)-(8) may be written.

$$
\begin{aligned}
& \left(1+\Delta t^{2}\right) x_{n+1}=x_{n}+\Delta t y_{n}+h \Delta t \Delta W_{n}, \\
& \left(1+\Delta t^{2}\right) y_{n+1}=y_{n}-\Delta t x_{n}+h \Delta W_{n} .
\end{aligned}
$$

Squaring, adding and taking expected values gives

$$
\mathbb{E}\left[x_{n+1}^{2}+y_{n+1}^{2}\right]=\frac{1}{1+\Delta t^{2}}\left(\mathbb{E}\left[x_{n}^{2}+y_{n}^{2}\right]+h^{2} \Delta t\right),
$$

and the result follows. 
Theorem 3.2 shows that given aribitrarily small $\Delta t>0$, BEM produces solutions with second moment that grows at a slower rate than the underlying SDE.

We now consider the following recurrence

$$
\begin{aligned}
x_{n+1} & =x_{n}+\Delta t y_{n}, \\
y_{n+1} & =y_{n}-\Delta t x_{n+1}+h \Delta W_{n} .
\end{aligned}
$$

The formula (9) for $x_{n+1}$ coincides with that for Euler-Maruyama (5), but the formula (10) for $y_{n+1}$ uses the new value $x_{n+1}$ in the right-hand side, rather than the value $x_{n}$ that appears in (6).

For deterministic systems in partitioned form, $\dot{u}=a(u, v), \dot{v}=b(u, v)$, there is a well known partitioned, or symplectic, Euler method; see, for example [2, Equations (1.9)]. For an SDE analogue, using the Euler-Maruyama stochastic increment leads to what we will call the Partitioned Euler-Maruyama (PEM) method. It can be shown that such a method shares the usual finite-time strong convergence properties of other Euler-based methods, of the type exemplified by [5, Corollary 10.6.4]. This follows from the general convergence theory in $[8,10]$, and a specific proof was given in [9], using a similar approach to that in $[4$, Appendix A].

It is appropriate to mention at this stage that a general framework for deriving symplectic methods for Hamiltonian systems with additive noise has been developed in [8]. Our work has the emphasis of showing the benefits of using a symplectic method via analysis on a simple test problem, and in this respect it closely matches section 6 of [8]. In [8, Proposition 6.2] a symplectic Euler-based method for a linear oscillator with additive noise is shown to have good meansquare error propagation over time intervals $[0, T]$, provided that $T \Delta t^{2}$ is small. Theorem 3.3 below deals with second moment behavior and applies in the $\Delta t$ fixed, $n \rightarrow \infty$ regime. The result shows that, unlike EM and BEM, the PEM recurrence gives excellent second moment growth properties.

Theorem 3.3. Consider the recurrence (9)-(10) arising from the Partitioned Euler-Maruyama method applied to the linear stochastic oscillator (1)-(2) with $x_{0}=1, y_{0}=0$. There exist functions $C_{\text {lower }}, C_{\text {upper }}:(0,2) \mapsto \mathbb{R}^{+}$such that for any $\Delta t<2$,

$$
C_{\text {lower }}(\Delta t)\left(1+h^{2} t_{n}\right) \leq \mathbb{E}\left[x_{n}^{2}+y_{n}^{2}\right] \leq C_{\text {upper }}(\Delta t)\left(1+h^{2} t_{n}\right), \quad \forall n \geq 0,
$$

where $C_{\text {lower }}$ and $C_{\text {upper }}$ are independent of $n$ with

$$
C_{\text {lower }}(\Delta t)=1+O(\Delta t), \quad C_{\text {upper }}(\Delta t)=1+O(\Delta t), \quad \text { as } \Delta t \rightarrow 0 .
$$


Proof. Suppose $\Delta t<2$. The method (9)-(10) may be written

$$
\begin{aligned}
{\left[\begin{array}{l}
x_{n} \\
y_{n}
\end{array}\right] } & =A\left[\begin{array}{l}
x_{n-1} \\
y_{n-1}
\end{array}\right]+\left[\begin{array}{c}
0 \\
h \Delta W_{n-1}
\end{array}\right] \\
& =A^{n}\left[\begin{array}{l}
1 \\
0
\end{array}\right]+\sum_{j=1}^{n} h \Delta W_{j-1} A^{n-j}\left[\begin{array}{l}
0 \\
1
\end{array}\right],
\end{aligned}
$$

where

$$
A=\left[\begin{array}{cc}
1 & \Delta t \\
-\Delta t & 1-\Delta t^{2}
\end{array}\right]
$$

We note that $A$ has eigenvalues $\lambda, \bar{\lambda}$ corresponding to eigenvectors $v, \bar{v}$, where

$$
\lambda=1-\frac{\Delta t^{2}}{2}+i \frac{\Delta t \sqrt{4-\Delta t^{2}}}{2}
$$

and

$$
v=\left[\begin{array}{c}
1 \\
-\frac{\Delta t}{2}+i \frac{\sqrt{4-\Delta t^{2}}}{2} .
\end{array}\right]
$$

The key to the success of the method is that $\lambda$ is of unit modulus.

Letting $\|\cdot\|$ denote the Euclidean vector norm, we have

$$
\mathbb{E}\left\|\left[\begin{array}{l}
x_{n} \\
y_{n}
\end{array}\right]\right\|^{2}=\left\|A^{n}\left[\begin{array}{l}
1 \\
0
\end{array}\right]\right\|^{2}+h^{2} \Delta t \sum_{j=1}^{n}\left\|A^{n-j}\left[\begin{array}{l}
0 \\
1
\end{array}\right]\right\|^{2} .
$$

We may expand

$$
\left[\begin{array}{l}
1 \\
0
\end{array}\right]=\alpha v+\overline{\alpha v}, \quad \text { where } \alpha=\frac{1}{2}-i \frac{\Delta t}{2 \sqrt{4-\Delta t^{2}}}
$$

Letting $\lambda=e^{i \theta}$ and $\alpha=|\alpha| e^{i \gamma}$, we find that

$$
A^{n}\left[\begin{array}{l}
1 \\
0
\end{array}\right]=2|\alpha|\left[\begin{array}{c}
\cos (\gamma+n \theta) \\
-\frac{\Delta t}{2} \cos (\gamma+n \theta)-\sin (\gamma+n \theta) \frac{\sqrt{4-\Delta t^{2}}}{2}
\end{array}\right] .
$$

It follows that

$$
\left\|A^{n}\left[\begin{array}{l}
1 \\
0
\end{array}\right]\right\|^{2}=1+\Delta t D(n, \Delta t),
$$

where $D(n, \Delta t)$ can be bounded uniformly in $n$; that is

$$
D(n, \Delta t) \leq \widehat{D}(\Delta t), \quad \text { for all } n .
$$


Further, there is a constant $\tilde{D}$ such that $\widehat{D}(\Delta t) \leq \tilde{D}$ for all sufficiently small $\Delta t$. Similarly we find that

$$
\left\|A^{k}\left[\begin{array}{l}
0 \\
1
\end{array}\right]\right\|^{2}=1+\Delta t E(k, \Delta t)
$$

where $E(k, \Delta t)$ can be bounded uniformly in $k$,

$$
E(k, \Delta t) \leq \widehat{E}(\Delta t), \quad \text { for all } k,
$$

and there is a constant $\tilde{E}$ such that $\widehat{E}(\Delta t) \leq \tilde{E}$ for all sufficiently small $\Delta t$.

The proof is completed by using (12) and (13) in (11).

\section{Oscillation Property of Partitioned Euler-Maruyama}

Theorem 3.3 shows that PEM captures the appropriate second moment growth given in Theorem 2.1. In this section we show that PEM also reproduces an exact analogue of the oscillation property in Theorem 2.2.

Theorem 4.1. Consider the recurrence (9)-(10) arising from the Partitioned Euler-Maruyama method applied to the linear stochastic oscillator (1)-(2) with $x_{0}=1, y_{0}=0$. For any $\Delta t<2$, the sequence $\left\{x_{n}\right\}_{n \geq 0}$ will switch signs infinitely many times as $n \rightarrow \infty$, almost surely.

Proof. Fix $\Delta t<2$. From (9)-(10), the sequence $\left\{x_{n}\right\}_{n \geq 0}$ from PEM satisfies

$$
X_{n}=B^{n} X_{0}+B^{n-1} r_{1}+B^{n-2} r_{2}+\cdots+r_{n}
$$

where

$$
X_{n}=\left[\begin{array}{c}
x_{n+1} \\
x_{n}
\end{array}\right], \quad r_{n}=\left[\begin{array}{c}
h \Delta t \Delta W_{n-1} \\
0
\end{array}\right], \quad B=\left[\begin{array}{cc}
\left(2-\Delta t^{2}\right) & -1 \\
1 & 0
\end{array}\right]
$$

and $X_{0}=[1,1]^{T}$. Considering the first component in (14), we get

$$
x_{n+1}=a_{n}+b_{n}+\sum_{i=1}^{n} a_{n-i} \hat{r}_{i}
$$

where $\hat{r}_{i}=h \Delta t \Delta W_{i-1} \sim N\left(0, h^{2} \Delta t^{3}\right)$ and the constants $a_{n}$ and $b_{n}$ satisfy $b_{n+1}=$ $-a_{n}$ and $a_{n+1}-\left(2-\Delta t^{2}\right) a_{n}+a_{n-1}=0$. 
Further algebra then reveals that there is a constant $K$ such that

$$
\left|a_{n}+b_{n}\right| \leq K
$$

and

$$
a_{n-i} \hat{r}_{i} \sim N\left(0, \sigma_{i}^{2}\right), \quad \text { with } \sigma_{i}^{2} \leq K h^{2} \Delta t^{3}
$$

Moreover,

$$
s_{n}^{2}:=\sum_{i=1}^{n} \sigma_{i}^{2} \rightarrow \infty \quad \text { as } n \rightarrow \infty .
$$

It follows that the Law of the Iterated Logarithm may be applied to the sequence $S_{n}:=\sum_{i=1}^{n} a_{n-i} \hat{r}_{i}$, see for example [1, Exercises 10.2, number 3]. We conclude that for any $\epsilon>0$ and $n$ sufficiently large, $S_{n}$ will almost surely exceed the bounds $-(1-\epsilon)\left(2 s_{n}^{2} \ln \ln s_{n}^{2}\right)^{1 / 2}$ and $(1-\epsilon)\left(2 s_{n}^{2} \ln \ln s_{n}^{2}\right)^{1 / 2}$ infinitely often. The boundedness of $a_{n}+b_{n}$ ensures that the same is true for $x_{n}$.

\section{Oscillation Properties of Strong Itô-Taylor Schemes}

In this section we give an oscillation theorem that is weaker than Theorem 4.1, but applies to a more general class of methods. The result requires only a finite time strong convergence condition.

Given a discrete approximation $\left\{x_{n}\right\},\left\{y_{n}\right\}$, we suppose that an interpolation procedure can be defined to give a continuous-time extension $\hat{x}(t), \hat{y}(t)$ with $\hat{x}\left(t_{n}\right)=x_{n}$ and $\hat{y}\left(t_{n}\right)=y_{n}$. Kloeden and Platen [5, Chapter 10] show how continuous-time extensions can be defined for the class of Itô-Taylor approximations and establish a general finite-time strong convergence theory. The following result is immediate. Note that the precise value of $\alpha$ is not important for Theorem 5.1.

Corollary 5.1 (Kloeden and Platen). For a strong Itô-Taylor approximation $\hat{x}(t), \hat{y}(t)$ of order $\gamma \geq 1 / 2$ applied to the linear stochastic oscillator (1)-(2) with initial data of bounded second moment, and with sufficiently small $\Delta t$, say $\Delta t \leq$ 1 ,

$$
\mathbb{E} \sup _{t \in[0, T]}\left\|\left[\begin{array}{l}
\hat{x}(t) \\
\hat{y}(t)
\end{array}\right]-\left[\begin{array}{c}
x(t) \\
y(t)
\end{array}\right]\right\|_{2} \leq C \Delta t^{\gamma},
$$

where the constant $C$ depends only on the initial data and $T$.

Proof. The SDE (1)-(2) satisfies the smoothness, global Lipschitz and linear growth requirements of [5, Corollary 10.6.4]. 
We will make use of the following lemma concerning the first zero-crossing time of $x(t)$. The key point in the lemma is that (18) holds independently of initial data. This allows us to prove an oscillation result for numerical methods by considering subintervals and making use of finite-time strong convergence.

Lemma 5.1. For the linear stochastic oscillator (1)-(2) with $x_{0}, y_{0} \in \mathbb{R}$, let $\tau_{1}$ denote the time of the first zero of $x(t)$ on $[0, \infty)$, that is,

$$
\tau_{1}=\inf \{t \geq 0: x(t)=0\}
$$

Then, uniformly in $x_{0}$ and $y_{0}$,

$$
\mathbb{P}\left(\tau_{1}>T\right)<\frac{2}{2^{T / \pi}} \quad \text { for each } T \geq \pi
$$

Proof. The proof is based on that of [6, Theorem 4.4, Chapter 8]. Here we allow for arbitrary initial data in $\mathbb{R}^{2}$.

We consider the nontrivial case $x_{0} \neq 0$. Recall that $x(t)$ is given by (3). Evaluate $x(t)$ at the discrete instants $t=k \pi, k=1,2, \ldots$, to obtain

$$
x(k \pi)=\cos (k \pi)\left[x_{0}-h \bar{B}(k \pi)\right], \quad \text { where } \bar{B}(k \pi)=\int_{0}^{k \pi} \sin s d W(s) .
$$

Hence $x(k \pi)>0$ if and only if

$$
\bar{B}(k \pi)\left\{\begin{array}{l}
>x_{0} / h \text { for } k=1,3, \ldots \\
<x_{0} / h \text { for } k=2,4, \ldots
\end{array}\right.
$$

Set

$$
Y_{k}=\int_{(k-1) \pi}^{k \pi} \sin s d W(s)
$$

so that $Y_{k} \sim N(0, \pi / 2)$ are i.i.d. and $\bar{B}(k \pi)=\sum_{i=1}^{k} Y_{i}$. Then, for $x_{0}>0$,

$$
\begin{aligned}
\left\{\tau_{1}>\pi\right\}=\{x(t)>0 \text { for all } 0 \leq t \leq \pi\} & \subset\{x(\pi)>0\} \\
& =\left\{\bar{B}(\pi)>x_{0} / h\right\} \\
& =\left\{Y_{1}>x_{0} / h\right\} \\
& =\left\{Y_{1}>\left|x_{0}\right| / h\right\} .
\end{aligned}
$$

For $x_{0}<0$ a similar argument gives

$$
\left\{\tau_{1}>\pi\right\} \subset\left\{-Y_{1}>\left|x_{0}\right| / h\right\} .
$$


Thus, for any $x_{0}$,

$$
\mathbb{P}\left(\tau_{1}>\pi\right) \leq \mathbb{P}\left(Y_{1}>\frac{\left|x_{0}\right|}{h}\right)=1-N\left(\frac{\left|x_{0}\right|}{h} \sqrt{\frac{2}{\pi}}\right)=: c\left(x_{0}, h\right),
$$

where $N(u)=(1 / \sqrt{2 \pi}) \int_{-\infty}^{u} e^{-y^{2} / 2} d y$. Furthermore, we have that for $x_{0}>0$

$$
\begin{aligned}
\left\{\tau_{1}>2 \pi\right\}=\{x(t)>0 \text { for all } 0 \leq t \leq 2 \pi\} & \subset\{x(\pi)>0, x(2 \pi)>0\} \\
& =\left\{\bar{B}(\pi)>x_{0} / h, \bar{B}(2 \pi)<x_{0} / h\right\} \\
& =\left\{Y_{1}>x_{0} / h, Y_{1}+Y_{2}<x_{0} / h\right\} \\
& \subset\left\{Y_{1}>x_{0} / h, Y_{2}<0\right\} .
\end{aligned}
$$

Similarly, for $x_{0}<0$ we find that

$$
\left\{\tau_{1}>2 \pi\right\} \subset\left\{-Y_{1}>\left|x_{0}\right| / h, Y_{2}>0\right\}
$$

Overall, for any $x_{0}$,

$$
\mathbb{P}\left(\tau_{1}>2 \pi\right) \leq \mathbb{P}\left(Y_{1}>\left|x_{0}\right| / h\right) \mathbb{P}\left(Y_{2}<0\right)=\frac{c\left(x_{0}, h\right)}{2} .
$$

Continuing this argument we find that

$$
\mathbb{P}\left(\tau_{1}>k \pi\right) \leq \frac{c\left(x_{0}, h\right)}{2^{k-1}} \text { for } k=1,2, \ldots
$$

In order to estimate the probability that $\tau_{1}$ exceeds an arbitrary positive number $T \geq \pi$, we let $\lfloor T / \pi\rfloor$ be the greatest integer not exceeding $T / \pi$, so $T-1<$ $\lfloor T / \pi\rfloor \pi \leq T$. Then

$$
\mathbb{P}\left(\tau_{1}>T\right) \leq \mathbb{P}\left(\tau_{1}>\lfloor T / \pi\rfloor \pi\right) \leq \frac{c\left(\left|x_{0}\right| / h\right)}{2^{\lfloor T / \pi\rfloor-1}}<\frac{4 c\left(x_{0}, h\right)}{2^{T / \pi}} .
$$

Since $c\left(x_{0}, h\right) \leq \frac{1}{2}$ uniformly in $x_{0}$ and $h$, the result follows.

We now state and prove the main result of this section. The theorem is weaker than Theorem 4.1- this is to be expected because it applies to a general class of numerical methods, including those that have been shown in Theorems 3.1 and 3.2 to have poor long-time second moment behavior. The theorem shows that by taking $\Delta t$ sufficiently small it is possible to guarantee any number of near sign changes with probability close to one. The proof uses only the finite-time strong convergence properties of the methods.

Theorem 5.1. Suppose that an Itô-Taylor scheme of strong order $\gamma \geq 1 / 2$ is applied to the linear stochastic oscillator (1)-(2) with $x_{0}=1, y_{0}=0$. Then given 
$\epsilon \in(0,1)$ and $N>0$ there exists a constant $\Delta t^{*}$ and a set of non-overlapping subintervals $S_{i}$, such that for all $\Delta t \leq \Delta t^{*}$

$$
\mathbb{P}\left(\left|\hat{x}\left(t_{i}\right)\right| \leq \epsilon \text { for some } t_{i} \in S_{i}, i=1,2, \ldots, N\right) \geq 1-\epsilon .
$$

Proof. Given $\epsilon$ and $N$, set $\Delta T=\pi \log _{2}\left(\frac{4 N}{\epsilon}\right)$ and take arbitrary non-overlapping subintervals $S_{i}=\left[\alpha_{i}, \beta_{i}\right]$ of length $\Delta T$. Note that $\Delta T \geq \pi$. Let $t_{i}$ denote the first time the true solution $x(t)$ crosses zero for $t \geq \alpha_{i}$. If the values $x\left(\alpha_{i}\right), \dot{x}\left(\alpha_{i}\right), i=$ $1,2, \ldots, N$, are known, we can regard the true solution on each interval as a new solution of (1)-(2) starting from initial values $x\left(\alpha_{i}\right), \dot{x}\left(\alpha_{i}\right)$. Hence, from Lemma 5.1, the probability that the true solution has at least one zero on $S_{i}$ satisfies

$$
\mathbb{P}\left(t_{i} \in S_{i}\right)=\mathbb{P}\left(t_{i} \leq \beta_{i}\right)>1-\frac{2}{2^{\Delta T / \pi}} .
$$

Let us denote by $A_{i}$ the event that the true solution crosses zero at least once on $S_{i}$, that is

$$
A_{i}=\left\{x\left(t_{i}\right)=0, t_{i} \in S_{i}\right\}
$$

Then we have

$$
\begin{aligned}
\mathbb{P}\left(x\left(t_{i}\right)=0 \text { for some } t_{i} \in S_{i}, i=1,2, \ldots, N\right) & =\mathbb{P}\left(A_{1} \cap A_{2} \cap \cdots \cap A_{N}\right) \\
& =\mathbb{E}\left[I_{A_{1}} I_{A_{2}} \cdots I_{A_{N}}\right]
\end{aligned}
$$

where $I$ denotes the indicator function. Letting $\mathcal{F}_{\alpha_{N}}$ be the sub- $\sigma$-algebra containing all information up to the point $\alpha_{N}$, and introducing conditional expectation, we get

$$
\begin{aligned}
\mathbb{E}\left[I_{A_{1}} I_{A_{2}} \cdots I_{A_{N}}\right] & =\mathbb{E}\left(\mathbb{E}\left[I_{A_{1}} I_{A_{2}} \cdots I_{A_{N}} \mid \mathcal{F}_{\alpha_{N}}\right]\right) \\
& =\mathbb{E}\left(I_{A_{1}} I_{A_{2}} \cdots I_{A_{N-1}} \mathbb{E}\left[I_{A_{N}} \mid \mathcal{F}_{\alpha_{N}}\right]\right) \\
& =\mathbb{E}\left(I_{A_{1}} I_{A_{2}} \cdots I_{A_{N-1}} \mathbb{E}\left[I_{A_{N}} \mid x\left(\alpha_{N}\right), \dot{x}\left(\alpha_{N}\right)\right]\right) \\
& >\left(1-\frac{2}{2^{\Delta T / \pi}}\right) \mathbb{E}\left[I_{A_{1}} I_{A_{2}} \cdots I_{A_{N-1}}\right] .
\end{aligned}
$$

Continuing this argument by introducing $\mathcal{F}_{\alpha_{N-1}}, \mathcal{F}_{\alpha_{N-2}}, \ldots$, and using $\frac{2}{2^{\Delta T / \pi}}<1$, we get

$\mathbb{P}\left(x\left(t_{i}\right)=0\right.$ for some $\left.t_{i} \in S_{i}, i=1,2, \ldots, N\right)>\left(1-\frac{2}{2^{\Delta T / \pi}}\right)^{N} \geq\left(1-\frac{2 N}{2^{\Delta T / \pi}}\right)$. 
Applying the Chebyshev inequality in (17), with $T:=\sup \left\{t: t \in S_{i}\right.$ for some $1 \leq$ $i \leq N\}$, we have

$$
\mathbb{P}\left(\sup _{t \in[0, T]}|\hat{x}(t)-x(t)| \geq \epsilon\right) \leq \frac{C \Delta t^{\gamma}}{\epsilon} .
$$

Equivalently,

$$
\mathbb{P}\left(\sup _{t \in[0, T]}|\hat{x}(t)-x(t)|<\epsilon\right) \geq 1-\frac{C \Delta t^{\gamma}}{\epsilon} \geq 1-\frac{C \Delta t^{* \gamma}}{\epsilon},
$$

for $\Delta t \leq \Delta t^{*}$, where $\Delta t^{*} \leq 1$ is yet to be defined.

Using (21) and (22), we have

$$
\begin{aligned}
\mathbb{P}\left(\left|\hat{x}\left(t_{i}\right)\right| \leq \epsilon \text { for some } t_{i} \in S_{i}, i=1,2, \ldots, N\right) \geq & \mathbb{P}\left(\left\{x\left(t_{i}\right)=0 \text { for some } t_{i} \in S_{i}, i=1,2, \ldots, N\right\} \cap\left\{\sup _{t \in[0, T]}|\hat{x}(t)-x(t)|<\epsilon\right\}\right) \\
\geq & \mathbb{P}\left(x\left(t_{i}\right)=0 \text { for some } t_{i} \in S_{i}, i=1,2, \ldots, N\right)+ \\
& \quad+\mathbb{P}\left(\sup _{t \in[0, T]}|\hat{x}(t)-x(t)|<\epsilon\right)-1 \\
\geq & \left(1-\frac{C \Delta t^{* \gamma}}{\epsilon}\right)+\left(1-\frac{2 N}{2^{\Delta T / \pi}}\right)-1 \\
= & 1-\frac{C \Delta t^{* \gamma}}{\epsilon}-\frac{2 N}{2^{\Delta T / \pi}} .
\end{aligned}
$$

Choosing

$$
\Delta t^{*}=\min \left(\left(\frac{2 N \epsilon}{C 2^{\Delta T / \pi}}\right)^{1 / \gamma}, 1\right),
$$

we find that the right-hand side of (23) is bounded below by $1-\epsilon$, as required.

Acknowledgement We thank Xuerong Mao for a number of helpful discussions on this topic.

\section{References}

[1] Y. S. Chow and H. Teicher. Probability Theory: Independence, Interchangeability, Martingales. Springer, 1978. 
[2] E. Hairer, C. Lubich, and G. Wanner. Geometric Numerical Integration. Springer, 2002.

[3] D. J. Higham. Mean-square and asymptotic stability of the stochastic theta method. SIAM J. Numer. Anal., 38(3):753-769, 2000.

[4] D. J. Higham, X. Mao, and A. M. Stuart. Exponential mean square stability of numerical solutions to stochastic differential equations. Mathematics Research Report 17, University of Strathclyde, 2002.

[5] P. E. Kloeden and E. Platen. Numerical Solution of Stochastic Differential Equations. Third Printing. Springer, 1999.

[6] X. Mao. Stochastic Differential Equations and Applications. Horwood, 1997.

[7] L. Markus and A. Weerasinghe. Stochastic oscillators. J. Differential Equations, 71(2):288-314, 1988.

[8] G. N. Milstein, Yu. M. Repin, and M. V. Tretyakov. Symplectic integration of Hamiltonian systems with additive noise. SIAM J. Numer. Anal., 39(6):2066-2088, 2002.

[9] A. H. Strømmen. Numerical Solution of Linear Stochastic Oscillators. Sivilingeniør thesis, Norwegian University of Science and Technology, 2001.

[10] L. B. Ryashko and H. Schurz. Mean square stability analysis of some stochastic systems. Dynam. Syst. Appl., 6(2):165-190, 1997.

[11] Y. Saito and T. Mitsui. Stability analysis of numerical schemes for stochastic differential equations. SIAM J. Numer. Anal., 33(6):2254-2267, 1996.

[12] J. M. Sanz-Serna and M. P. Calvo. Numerical Hamiltonian Problems. Chapman \& Hall, 1994.

[13] H. Schurz. The invariance of asymptotic laws of linear stochastic sytems under discretization. Z. Agnew. Math. Mech., 79:375-382, 1999.

[14] H. Schurz. Moment attractivity, stability and contractivity exponents of stochastic dynamical sytems. Disc. Cont. Dyn. Syst., 7:487-515, 2001. 